\title{
A 6LoWPAN Routing Optimization Mechanism for WMSN
}

\author{
Jing Li, ${ }^{1}$ Chong Shen, ${ }^{1}$ and Kae Hsiang Kwong ${ }^{2}$ \\ ${ }^{1}$ College of Information Science \& Technology, No. 58, Renmin Avenue, Haikou, Hainan Province 570228, China \\ ${ }^{2}$ Malaysian Institute of Microelectronic Systems, Technology Park Malaysia, 57000 Kuala Lumpur, 999004, Malaysia
}

Correspondence should be addressed to Chong Shen; chongshen@hainu.edu.cn

Received 1 February 2013; Accepted 3 March 2013

Academic Editors: W. Gao and S. Ozdemir

Copyright (C) 2013 Jing Li et al. This is an open access article distributed under the Creative Commons Attribution License, which permits unrestricted use, distribution, and reproduction in any medium, provided the original work is properly cited.

\begin{abstract}
In Wireless sensor networks (WSNs), group mobility is important in many practical application scenarios, and it is inconvenient for traditional static WSNs to collect information. In this paper, we propose a WSN model based on IPv6 over Low power Wireless Personal Area Networks (6LoWPANs) in mobile sensing application scenarios. Wireless mesh sensor network (WMSN) infrastructure and Network Mobility (NEMO) protocol are deployed to ensure the continuity of the communications. The routing optimization mechanism in the nested network is then discussed. By taking mobile network partition and IP address configuration in the nested network, the corresponding signaling flow is discussed. Simulation results indicate that our mechanism is able to minimize the transmission costing, handoff delay, throughput and energy consumption of sensor nodes. Energy of each mobile router (MR) saves around $0.2 \mathrm{~J}$ per 3 seconds.
\end{abstract}

\section{Introduction}

With the maturation of wireless sensing technologies, the mobile sensing communication systems are widely applied and deployed on a global scale. The demand for information and mobility businesses hopes that some of the fixed and mobile nodes in wireless sensor network are composed of a collection of subnets as a whole, while the whole can move and access the Internet seamlessly. It is urgent that we need mobility mechanisms to support the mobile of a single terminal and subnet terminal. For example, mobile personal area networks where the sensor networks are deployed in vehicular objectives such as aircraft, automobiles, and trains [1].

The development of traditional WSN mobility management technology is still not mature enough; most mobility protocols of traditional wireless sensor networks only aim at coping with weak mobility, such as join a new node in WSNs or make a node in WSNs invalid, and it cannot completely solve problems with strong mobility. However, with 6LoWPAN-based IPv6-WSN proposal [2], IPv6-based wireless sensor network mobility management scheme is developing. 6LoWPAN [3] working group was created by Internet Engineering Task Force (IETF). 6LoWPAN protocol became a standard in 2006. The standard specifies an adaption layer above IEEE 802.15.4 MAC and Routing layers to support IPv6 packets' transmission over IEEE 802.15.4, and network mobility can therefore be supported in WSNs.

NEMO can solve problems of network mobility. At the same time, it has the higher efficiency in broadband resource utilization and better service quality assurance. The largest characteristic of NEMO is that when the topology of a network changes, the location will update through MR and complete the whole handoff to avoid a large number of the mobile network nodes (MNNs) simultaneous care-ofaddress configuration and location update in the subnet. NEMO greatly reduces the handoff frequency of the network, increases utilization of network bandwidth and coverage, optimizes network overhead from mobility management protocol, enhances network scalability, and improves the network operation procedures.

At present, the research for IPv6 subnet mobility is lacking fundamental studies. A lot of technical details need to be further improved. Routing optimization issues include nested multilayer, multilink, safety, reliability, and many other internal problems; those problems greatly limit the practical deployment of the network. Therefore, the network mobility is necessary to be explored to solve the current problems 
of NEMO and to achieve further performance optimization of mobility management technology; it is urgent to propose novel routing algorithms for next generation IPv6 sensor networks.

As a great extension to the ad hoc network, wireless mesh network (WMN) [4] is becoming an important mode. All sensor nodes in the WMSN have planned routes to base stations, so the network has a good performance on fault recovery. In addition, regarding the power saving, each sensor node data transmission is decreased by using multihop instead of single hop. Above all, this paper proposes a new algorithm which applies WMSN structure into group mobility. We analyze the data transmission process and transmission rate as well as energy consumption in the stationary and mobile sensor networks. When there are nested mobile networks, through the adoption of the mobile network partition and IP address configuration, we prove that the algorithm can effectively reduce handoff delay and energy consumption of sensor nodes. At the same time, the encapsulation costing and "Ping-Pong" routing problem also can be optimized.

In $[5,6]$, we have proposed the IPv6 enabled mobility framework to improve the user mobility experience. Using NEMO we can provide a flexible network integration mechanism across Wi-Fi, WiMax, and UMTS systems vertically. The flexible approach overcomes the limitation of different networks access denial when nodes move using two operations: Policy enforced handover management and Dynamic handover implementation. NEMO has been deployed to enable WSN with improved mobility experience [7]. This paper is an extension of our previous work which focuses on network mobility routing optimization mechanism to reduce the nested network "Ping-Pong" routing issue.

The paper starts with a short description on the state of 6LoWPAN and communication mechanism of NEMO protocol in Section 2. Section 3 details our new mobility framework based on 6LoWPAN and the communication of the routing mechanism in the nested network using mobile network partition and IP address configuration. We verify that our proposed routing mechanism on delay performance, transmission overhead, throughput, and the node energy consumption has a better performance than NEMO and ROTIO in Section 4, and finally we detail our research conclusion in Section 5.

\section{Related Works}

There have been many research efforts on NEMO and group mobility. In this section, we review the state-of-the-art research and work related issues with the focuses on routing optimization mechanism in WSNs.

6LoWPAN employs the PHY and MAC layer protocols defined in IEEE802.15.4, while the upper protocol in network layer is the IPv6 protocol. To support seamless data transmission between MAC layer and network layer, a network adaptation layer between them is introduced by 6LoWPAN working group. The adaptation layer is responsible for the fragmentation, reassembly of fragments, IPv6 header compression, and handling of the mesh addressing and broadcast headers. In the field of safety monitoring applications, IPbased WSNs are more robust and can timely and effectively monitor the occurrence of disasters in order to reduce losses. IP technology is of great significance in WSNs.

As an extension of mobile IPv6 (MIPv6) protocol, NEMO [8] is standardized by IETF, used to support the network mobility; mobile router (MR) can provide a network connection for mobile network. NEMO protocol is based on the development of mobile IP technology. The NEMO network is composed of mobile router, home address (HA), and MNNs. The communication between all MNNs and Correspondent Node $(\mathrm{CN})$ is completed through a bidirectional tunnel between MR-HA and MR. Compared with other mobile network technologies, NEMO has obvious advantages in bandwidth, cost, and so on. The network, which MR and MNNs initially register when they are not moving, is marked as their home network. While MR and MNNs are attached to their home network, MR is assigned a home address (MR-HA), and MNNs are assigned IP addresses with a common mobile network prefix (MNP). When MR with the mobile nodes moves into a foreign network, the MR will configure a care-of-address (CoA) through receiving the route advertising (RA) message from the Internet access router (AR), and then MR informs the new CoA and MNP to its MR-HA. Two bindings; that are between new CoA and MR-HA and between MNP and MR-HA can then be set up, and a bidirectional tunnel between MR-HA and MR is established. When the packet destined for MNNs arrives at the home network, the MR-HA intercepts it and sends it to the mobile router with new position [9].

When nested mobile networks are created, the data transmission needs to traverse each MR and their home agent. It can easily cause the "Ping-Pong" routing and packet loss. Therefore, to solve routing optimization problems in a nested network, a variety of routing optimization schemes in the mobile network have been proposed. For example, the routing optimization using tree information option (ROTIO) [10], Access Router Option (ARO) [11]. In the ROTIO scheme, MR in the mobile network must make binding update twice; as nesting depth increases, the overhead of binding update will be increased, and the toplevel mobile router needs a lot of storage space in the binding cache to store binding information; the storage overhead is therefore increased. In ARO, authors propose they can add a new ARO access router it can eliminate tunnel package between the parent MR of MR and home agent in nested NEMO.

\section{A Routing Scheme for Network Mobility of WSN Based on 6LoWPAN}

In this section, we mainly describe integration of WMSN with NEMO based on 6LoWPAN, and the new nested routing optimization mechanism (NNRO) is proposed. 


\subsection{Integration of NEMO with WMSN Based on 6LoWPAN}

3.1.1. Network Architecture. The architecture of WMSN is composed of mesh routers and wireless sensor nodes. WSNs are reconstructed into mesh networking topologies. To interconnect IP-based network and WSNs seamlessly, we employ the 6LoWPAN IP address configuration in wireless sensor nodes where sensor nodes are able to access to AR directly. In order to solve the problem of the group mobility in WSNs, we introduce the NEMO protocol at the network layer. Figure 1 shows our architecture to support the integration of WMSN and NEMO protocol based on 6LoWPAN.

In Figure 1, the architecture is divided into three layers: A: data acquisition layer, B: data transmission layer, C: internet layer where users acquire information through the Internet. A wireless sensor network is divided into a number of subnetworks, that is, several clusters where mesh router directly communicates with cluster heads. The cluster head sends/receives messages to/from sensor nodes. Every mesh router connects to the Internet through the AR. When one of the subnetworks leaves its home link, the others will automatically form a new network topology. Once subnetwork moves, mesh router will play the original role of MR according to NEMO protocol. The existence of multiple MRs improves the robustness of WSNs performance.

Following NEMO standards, the MR is responsible for mobility management of mobile terminals inside the group to apply NEMO in 6LoWPAN-based WSN. In WMSN, when an MR loses efficiency, the most powerful node in the subnetwork is selected as the cluster head to play the MR role. Through communicating with an MR or multiple MR mobile sensor nodes inside the mobile network can access the Internet and transmit IP-based packets to/from the Internet when they are moving.

In other scenarios, when a cluster in the sensor network moves to some specific positions, MR in this cluster can play the same role with the other mobile nodes. If the location of mobile network is fixed, MR plays a regular router role with fixed routing services.

3.1.2. The Communication Processing. When an MR is in the home network, it receives neighbor broadcast messages from $\mathrm{AR}$ periodically. If the $\mathrm{MR}$ does not receive the information from AR during a prescript time, MR will send a routing request message to $A R$. If $A R$ does not respond, it is confirmed that MR and the group mobility are in the coverage of another AR; the MR continues to broadcast routing request messages until it receives neighbor broadcast messages from a new AR. The MR will configure CoA based on the network prefix information in the received messages.

The MR sends binding update (BU) message to home agent. The BU message includes mobile network prefix and home address. When MR-HA receives the BU message, it will update the routing table and establish the mapping between MR-HA and MR. The source address is the home agent address, and destination address is the access router address. Home agent sends the binding acknowledgment message to the mobile router. If this operation is successful, home registration process is regarded as a success. Then, sensor nodes can communicate with IPv6 Internet hosts successfully.

3.2. The Routing Mechanism in the Nested Network. When we employ traditional NEMO agreement in the nested mobile network, the data packets must go through each MR and their home agent, the data package is sent through the tunnel between HA and MR. Therefore, data packets are encapsulated at each MR of NEMO paths (the red line in Figure 2) [12]. The problems here are that the encapsulation can easily cause multiple packaging, "Ping-Pong" routing, and packet loss. Meanwhile, the encapsulation processing increases network responding time, congests data transmitting layers, and also increases the internetwork/internetwork handoff delay.

3.2.1. Routing in the NEMO. In NNRO, all MRs of the NEMO configure a CoA with topological significance through Dynamic Host Configuration Protocol (DHCP) in WMSN based on 6LoWPAN [13]; they are directly registered to $\mathrm{CN}$. Multiple MRs form a tree hierarchy in which the root MR is called the top-level MR (TLMR). Each MR has IPv6 addresses which connects to TLMR through the IPv6 address and provides host routes in nested NEMO internally. The data packets are sent to the authorized IPv6 address of the NEMO through the route. The new processes avoid multiple encapsulation of data packets while reducing the communication complexity between two sensor nodes in the same mobility group.

In addition, we provide mobile access router (MAR) [14] for the nested network to complete the transformation of DHCP request [15]. The specific process is shown in Figure 3: TLMR with TLMR-CoA configuration is connected to AR. MR2 is connected to an MAR. The MAR configures a CoA in MR2 external interface which belongs to NEMO1. When MR2 sends an IPv6 request, the MAR will send this request to TLMR, and then TLMR sends a request to AR for getting an IPv6 address; the IPv6 address is assigned to MR2 through the MAR. In the end, TLMR will add the host route of the address to its own routing table.

3.2.2. Handoff between Different ARs. When MR3 moves into the network managed by MR2, MR3 will get CoA1 in a foreign link. Here, we consider the case that MR3 moves into the network managed by MR1 after leaving MR2 managed network. When MR1 is aware of accessing the network managed by MR3, it informs MR3 to get another CoA2 address. If MR1 and MR2 belong to different access networks, the MR3 gets a new IPv6 address through address grant mechanism. However, when MR1 and MR2 belonging to the same access network, through the same process described above, the process can largely increase the handoff time.

So we put forward partition updated scheme between different ARs. The scheme is that the movement of MR and MNNs belong to the same AR is called the movement in the same district, and the movement between different ARs is called the interval movement. As long as the movement is in the same AR, it does not need to register to its home agent. 


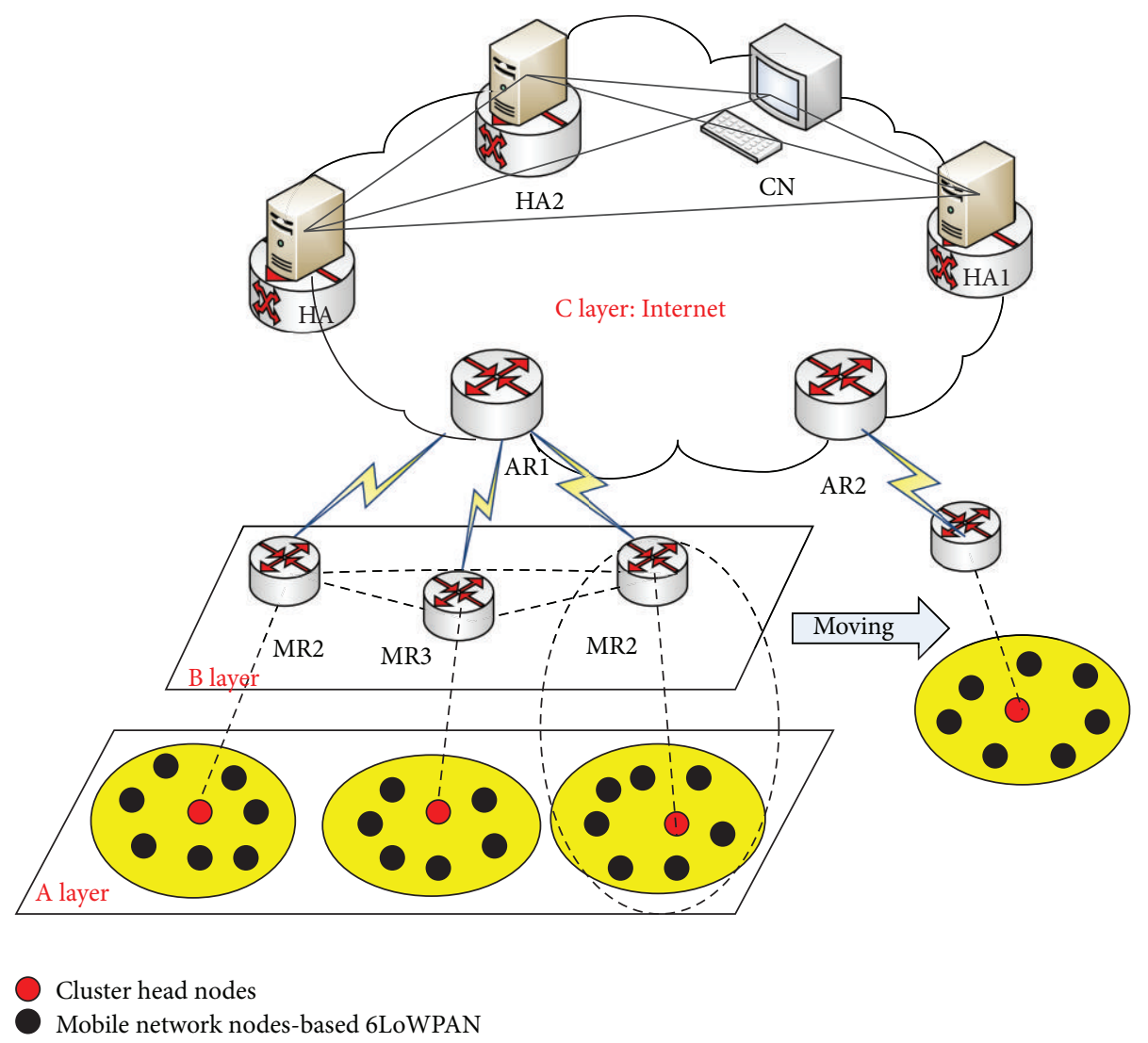

FIGURE 1: The architecture of WMSN based on 6LoWPAN.

When the movement is in different ARs, the MR needs to register to MR-HA, and then obtains a new IPv6 address from new AR.

The specific handoff process is as follows (Figure 4): MR2 is managed by an AR. When MR3 enters the managed network of MR2, MR3 gets two addresses that are CoA1 and CoA2. CoA1 is known as the care-of address inside the region, and CoA3 is a care-of address of the foreign link, which is known as the interval care-of address. Then, MR3 sends two $\mathrm{BU}$ messages, one is sent to MR3-HA in order to register CoA2, and the other is sent to local access router in order to register the care-of address CoA1 and CoA2. Two care-of addresses will be bound together through $\mathrm{AR}$, and the packets whose destination is CoA 2 are sent to CoA1. So, if MR 3 moves in the same area (such as the area managed by AR1), it does not need to register to home agents. If MR3 moves to the MR1, it needs to configure a new CoA3 and sends BU to AR1. When AR1 receives $\mathrm{BU}, \mathrm{AR} 1$ builds a binding between CoA3 and CoAl and removes the binding between CoAl and CoA2. Thus, the handoff processing is completed.

3.2.3. Routing outside the NEMO. According to the traditional NEMO protocol, the sending of data packets needs to go through all MR's home agents (the red line shown in Figure 2). To improve the routing performance, in NNRO, when the mobile nodes need to send data packets, packets are sent to an MR first and then encapsulated. After experiencing the upper MRs in the nested tree, encapsulate packets reach the management of AR. Because MRs have a unique IPv6 address, the upper MRs send packets without any encapsulation costing. When packets reach the management of an AR from upper MR, The packets after decapsulation will be analyzed through AR to know the destination of packets, and then the packets will be sent to $\mathrm{CN}$. Through this way, it does not need to go through any home agent; it also reduces the expenses of encapsulation and improves the data rate of transmission.

\section{Simulation Results and Analysis}

4.1. Simulation Scenario. In this section, the comparison shows that the performance of the new proposed routing mechanism is better than the performance of ROTIO and NEMO basic protocol on delay performance, transmission overhead, throughput, and the node energy consumption. Using network simulator 2 (NS-2), we measured transmission costing, the handoff delay, throughput, and energy saving performance. The wireless link works at $2.4 \mathrm{GHz}$ with $250 \mathrm{kbps}$ data rate. Mobile subnetwork and the nodes of mobile subnetwork are assumed to move with the same speed 


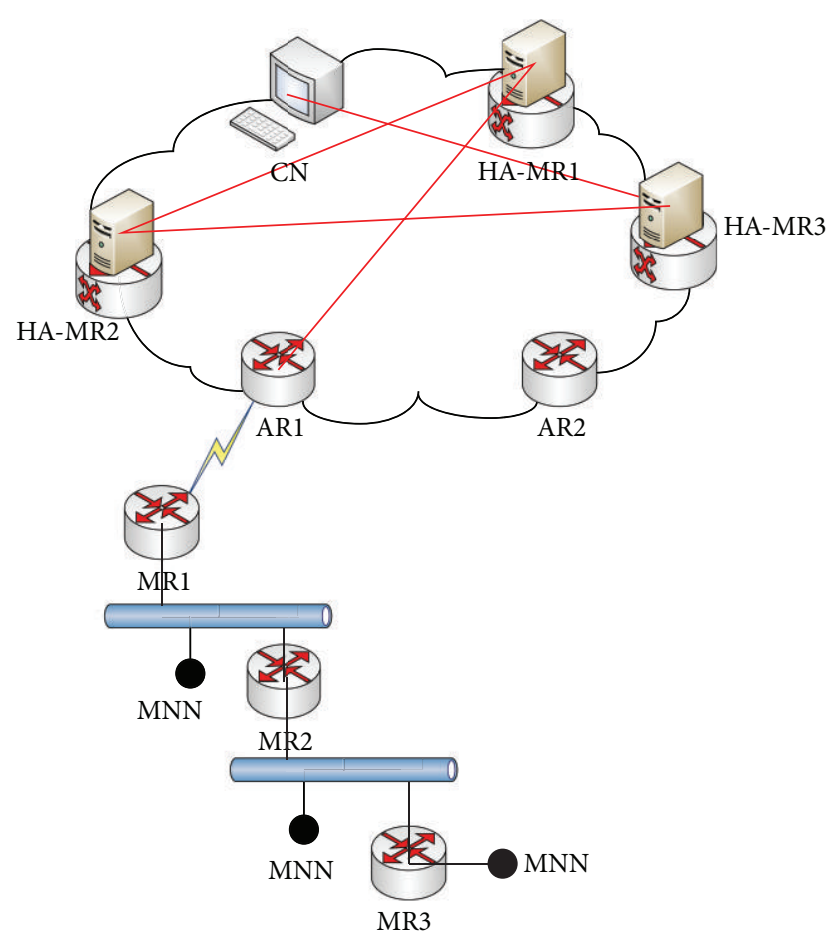

FIgURE 2: The traditional nested NEMO.

TABLE 1: Parameter for Simulation.

\begin{tabular}{lc}
\hline Parameter & Values \\
\hline Simulation area size & $300 \mathrm{~m} \times 300 \mathrm{~m}$ \\
Total time of simulation & $100 \mathrm{~s}$ \\
Node transmission range & $20 \mathrm{~m}$ \\
Node velocity & $3 \mathrm{~m} / \mathrm{s}$ \\
Transmission range & $10 \mathrm{~m}$ \\
Initial node energy & $10 \mathrm{~J}$ \\
Energy consumption during transmission & $0.03 \mathrm{~J}$
\end{tabular}

along the same direction. Using AODV routing protocol as traditional routing protocol, MR network takes the shape of mesh network by adapting AODV or LOAD routing protocol in the new routing mechanism. Data stream is selected as constant bit rate (CBR); each mobile router includes five mobile network nodes. Other parameters for simulation are stated in Table 1.

4.2. Simulation Analysis. In Figure 5, the total transmission costing of three schemes increases varying numbers of network nested levels. When nesting NEMO level is large, the total transmission costing of NEMO protocol is much larger than the other schemes, and the total transmission costing of NNRO scheme is minimal. When the nesting level is increasing, the growth rate of the total transmission costing of NNRO scheme is the slowest. Because the time of encapsulation is needed only one in the NNRO scheme, it reduces unnecessary packaging overhead. In the NNRO scheme, transmission path of the registration packet is also

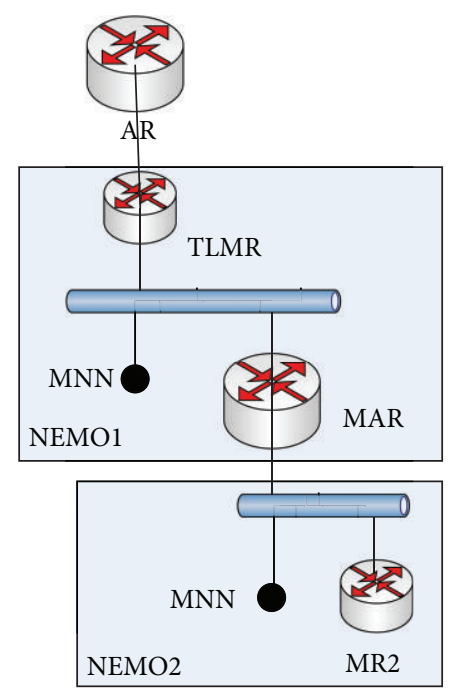

FIGURE 3: Routing in the NEMO.

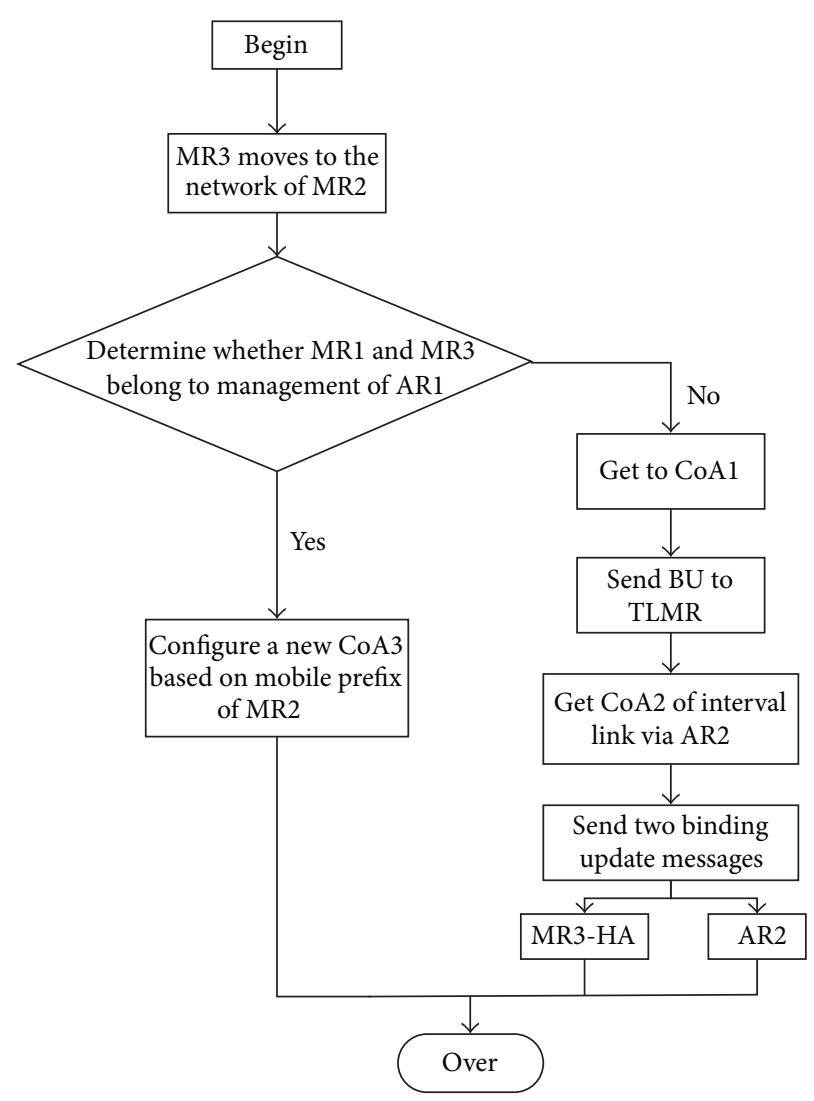

FIgURE 4: Handoff between different ARs.

the shortest, because MRs send message to the cluster head. Firstly, the cluster head broadcasts information after analysis, and then the cluster head finds an optimum routing in the router to send the message.

Figure 6 shows the handoff delay experienced by constant bit rate traffic rate 10 packets per second. In Figure 6 , the handoff delay of NNRO scheme is minimal, the average 


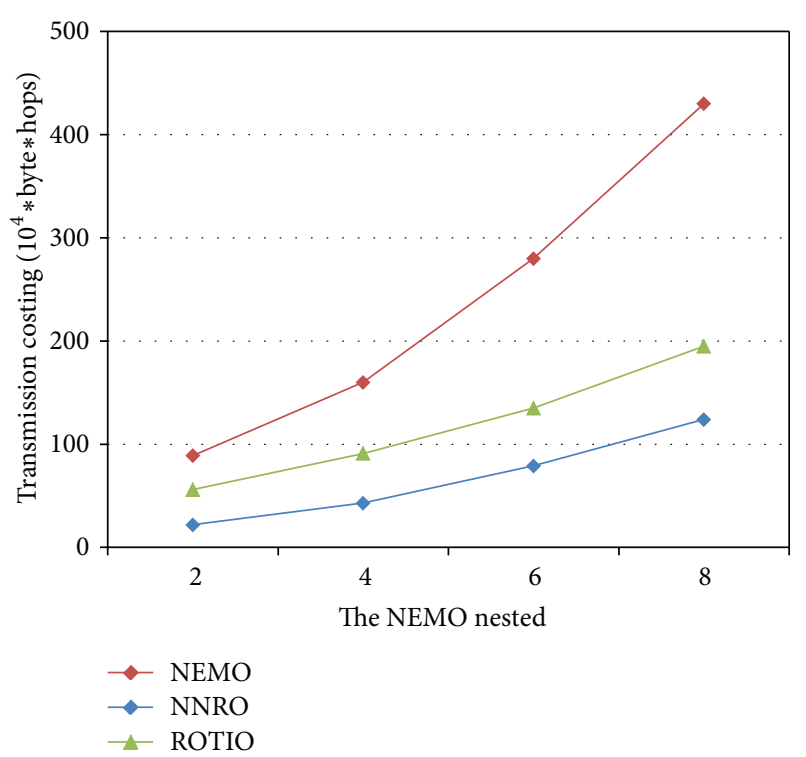

FIGURE 5: With the nested level increasing, the changing of transmission costing.

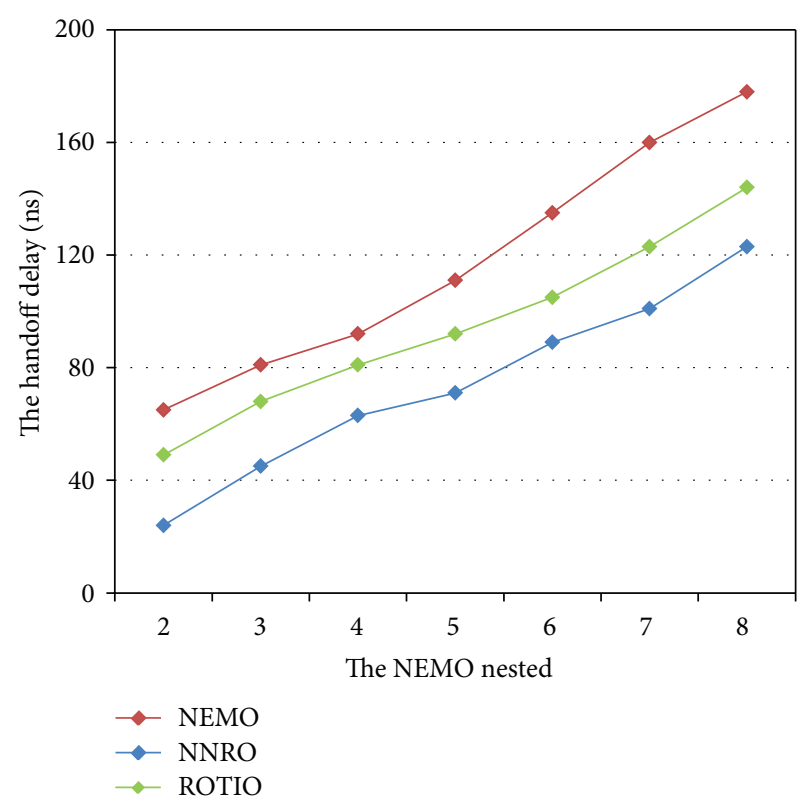

Figure 6: The time of the handoff delay.

handoff delay of ROTIO scheme and NNRO scheme is less than the NEMO basic protocol, because in the internal handoff the packet transmission path is only internal NEMO, the data of handoff delay is relatively small, as the nesting depth increases, the growth rate of handoff delay of the NEMO protocol is faster than ROTIO and NNRO, and the growth rate of the handoff delay of the NNRO scheme is the lowest. In the NNRO scheme, encapsulation of data packets is only once, and in ROTIO, the encapsulation of data packets is needed twice. We propose a kind of partition updated scheme, which reduces the times of registration to HA. As

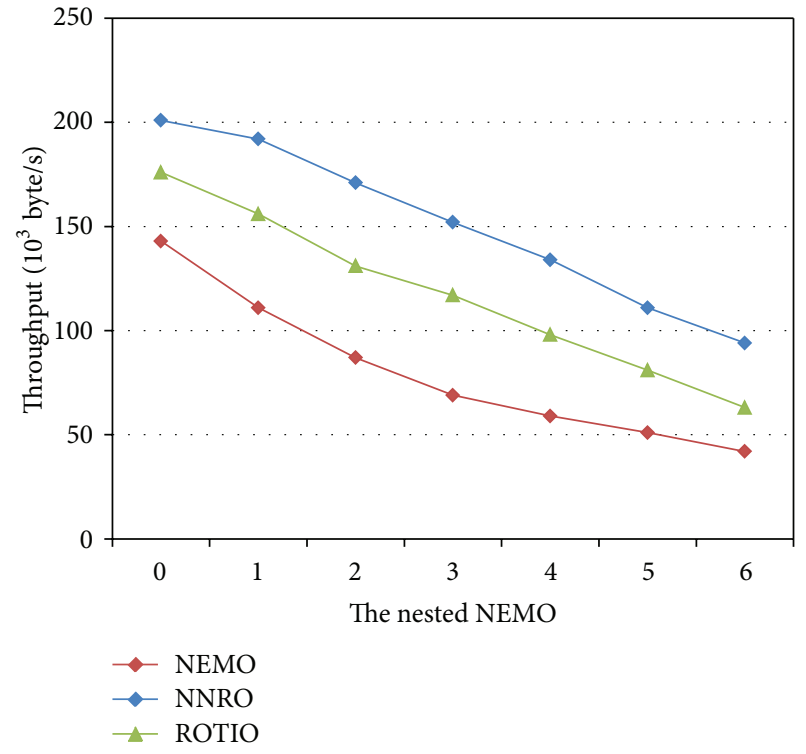

FIGURE 7: The changing of throughput.

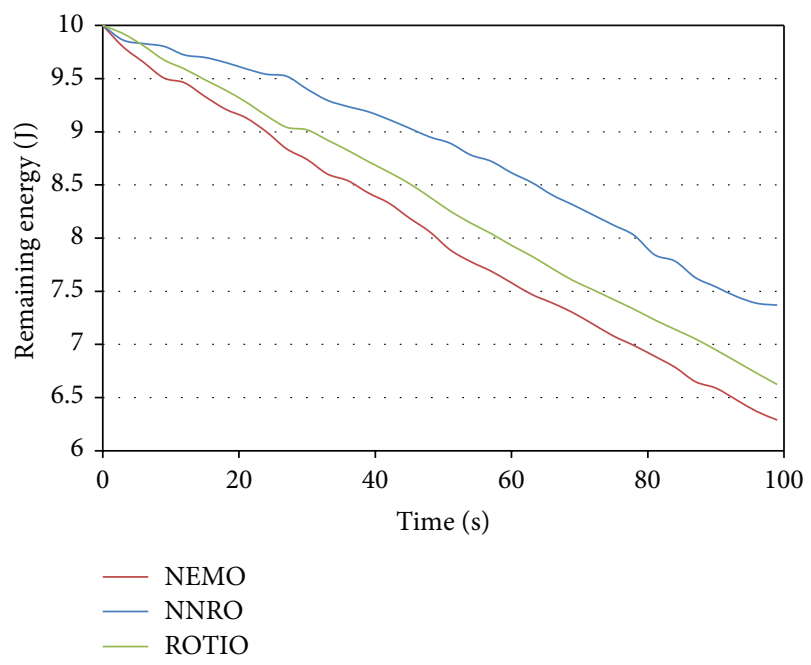

Figure 8: The remaining energy as a function of simulation time.

long as all of the movements belong to the management of an $\mathrm{AR}$, it does not need to register to its home agent. When the movement is in different ARs, the MR needs to register to MR-HA, and then obtains a new IPv6 address from new AR. The above result proves that the new mechanism performs better in the handoff delay.

The transmission rate of the communication packet data sets 300 packets per second in the simulation, and Figure 7 shows a throughput changing varying numbers of network nested levels. The throughput of the three schemes is all reducing; the reason is that transmission delay and handoff delay are both increasing, and they impact on the throughput. The NNRO scheme is superior to the ROTIO scheme. Because taking mobile network partition in the nested network is proposed. The partition mechanism reduces the 
unnecessary hometown register and handoff delay. Compared to traditional NEMO, the throughput in the improved routing mechanism reduces slowerly.

The node's initial energy is set $10 \mathrm{~J}$. Figure 8 shows the remaining energy as a function of simulation time. With the time increasing, the remaining energy of every node decreases gradually. Compared to the traditional NEMO network, it reduces encapsulation of data packets. Using traditional NEMO in the nested mobile network, the packet must go through each MR and its home agent, and between $\mathrm{MR}$ and HA the packet must be encapsulated; therefore, the packet will be encapsulated at each MR in the NEMO. In NNRO, we propose an IP address configuration, and MRs register directly to $\mathrm{CN}$. Each MR has an IPv6 address accessed by TLMR and provides host routing in nested NEMO, so the packet can be forwarded through the routing to the IPv6 address. It can achieve routing available in the nested network, avoiding multiple package of the packet. So the improved routing mechanism is better than the traditional NEMO network on the energy consumption. Because we utilize the partition mechanism to our mobile network, the partition mechanism reduces the unnecessary hometown register and obtained of care-of-address; thus, the remaining energy for ROTIO mobility management scheme is much less than that for NNRO-based method.

\section{Conclusions}

This paper proposed a 6LoWPAN routing optimization mechanism for WMSN. By taking mobile network partition and handoff delay consideration, MR reduces the number of obtained care-of address. IP address configuration into the group mobility in WMSN effectively reduces the data package, and communication between the two nodes in the same nested network. We show that comparing the state-ofthe-art algorithms and newly proposed scheme, the scheme offers less energy consumption, reduced handoff delay, and higher throughput. The scheme also resolves "Ping-Pong" routing problems, which are highly desirable for energy and computation capability limited sensor nodes.

\section{Acknowledgments}

The authors gratefully acknowledge the financial support of China International Science and Technology Cooperation Project (no. 2013DFR11020) and also would like to thank the support of Hainan International Science and Technology Cooperation Project (no. 2012-GH008). Thanks are due to Dr. Wencai Du who gives much contribution to the paper.

\section{References}

[1] J. B.-Y. Liu, P. Brass, O. Dousse, P. Nain, and D. Towsley, "Mobility improves coverage of sensor networks," in Proceedings of the 6th ACM International Symposium on Mobile Ad Hoc Networking and Computing (MobiHoc '05), pp. 300-308, 2005.

[2] C. Perkins, D. Johnson, and J. Arkko, "Mobility Support in IPv6," IETF RFC, 6275, July 2011.
[3] N. Kushalnagar and G. Montenegro, "6LoWPAN: overview, assumptions problem statement and goals," draft-ietf-6lowpanproblem-08 (work in progress), February 2006.

[4] K. Rayner, "Mesh wireless networking communications engineer," Communications, vol. 1, no. 5, pp. 44-47, 2003.

[5] C. Shen, W. Du, R. Atkinson, J. Irvine, and D. Pesch, "A mobility framework to improve heterogeneous wireless network services," International Journal of Ad Hoc and Ubiquitous Computing, vol. 7, no. 1, pp. 60-69, 2011.

[6] C. Shen, W. Du, R. Atkinson, and K. H. Kwong, "Policy based mobility \& flow management for IPv6 heterogeneous wireless networks," Wireless Personal Communications, pp. 1-33, 2010.

[7] C. Shen and W. Du, "Mobility modeling for vehicular communication data dissemination," in Computer and Information Science 2010, vol. 317 of Studies in Computational Intelligence, pp. 35-45, Springer, Berlin, Germany, 2010.

[8] V. Devarapalli, R. Wakikawa, A. Petrescu et al., "Network Mobility (NEMO) Basic Support Protocol," IETF RFC3963, 2005.

[9] R. Chai, Y. L. Zhao, Q. B. Chen, T. Dong, and W. G. Zhou, "Group mobility in 6LoWPAN-based WSN," in Proceedings of International Conference on Wireless Communications and Signal Processing (WCSP '10), October 2010.

[10] H. Cho, T. Kwon, and Y. Choi, "Route optimization using tree information option for Nested mobile networks," IEEE Journal on Selected Areas in Communications, vol. 24, no. 9, pp. 17171724, 2006.

[11] C. Ng, "Securing Nested Tunnels Optimization with Access Router Option," IETF, draft-ng-nemo-access-router-option-01, July 2004.

[12] M. Calderón, C. J. Bernardos, M. Bagnulo, I. Soto, and A. de la Oliva, "Design and experimental evaluation of a route optimization solution for NEMO," IEEE Journal on Selected Areas in Communications, vol. 24, no. 9, pp. 1702-1716, 2006.

[13] M. Hasan, A. H. Akbar, H. Mukhtar, K. H. Kim, and D. W. Kim, "A scheme to support mobility for IP based sensor networks," in Proceedings of International Conference on Software Testing (ICST '08), pp. 1-9, 2008.

[14] C. J. Bernardos, M. Bagnulo, and M. Calderón, "MIRON: MIPv6 route optimization for NEMO," in Proceedings of the 4th Workshop on Applications and Services in Wireless Networks (ASWN '04), pp. 189-197, August 2004.

[15] G. Tsirtsis, H. Soliman, N. Montavont, G. Giaretta, and K. Kuladinithi, "Flow Bindings in Mobile IPv6 and Network Mobility (NEMO) Basic Support," IETF, RFC, 6089, January 2011. 

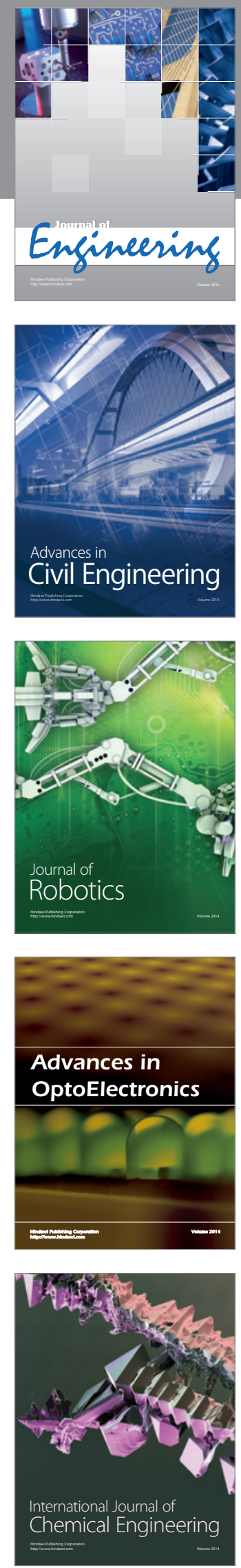

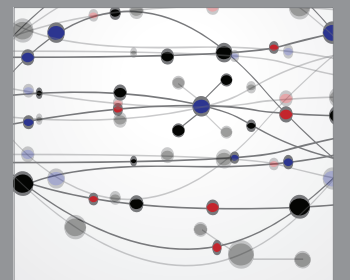

The Scientific World Journal
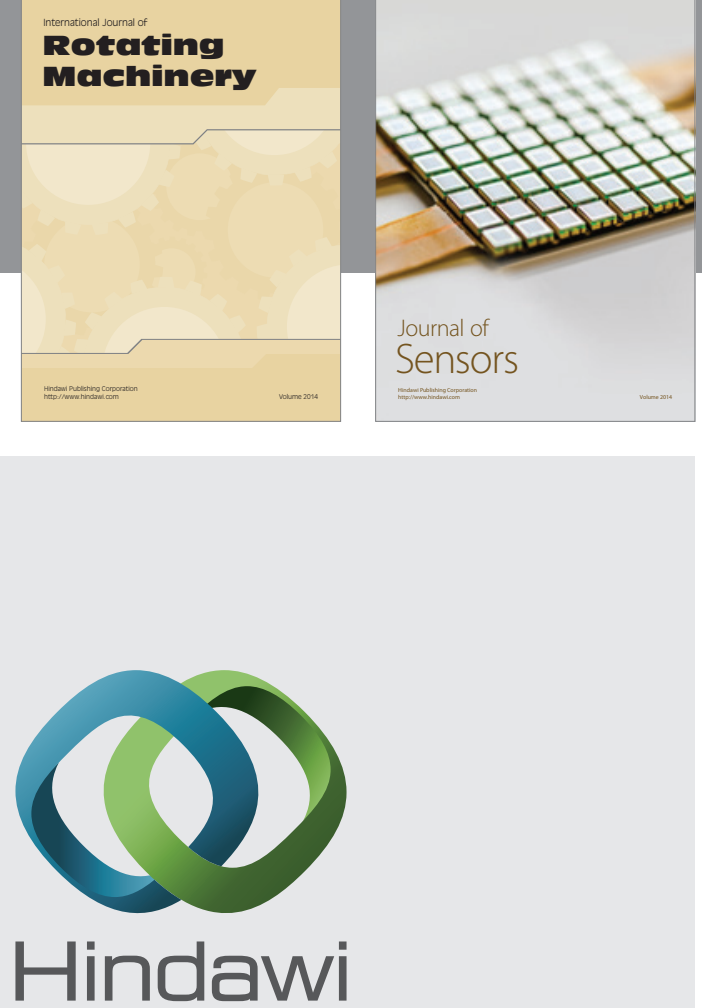

Submit your manuscripts at http://www.hindawi.com
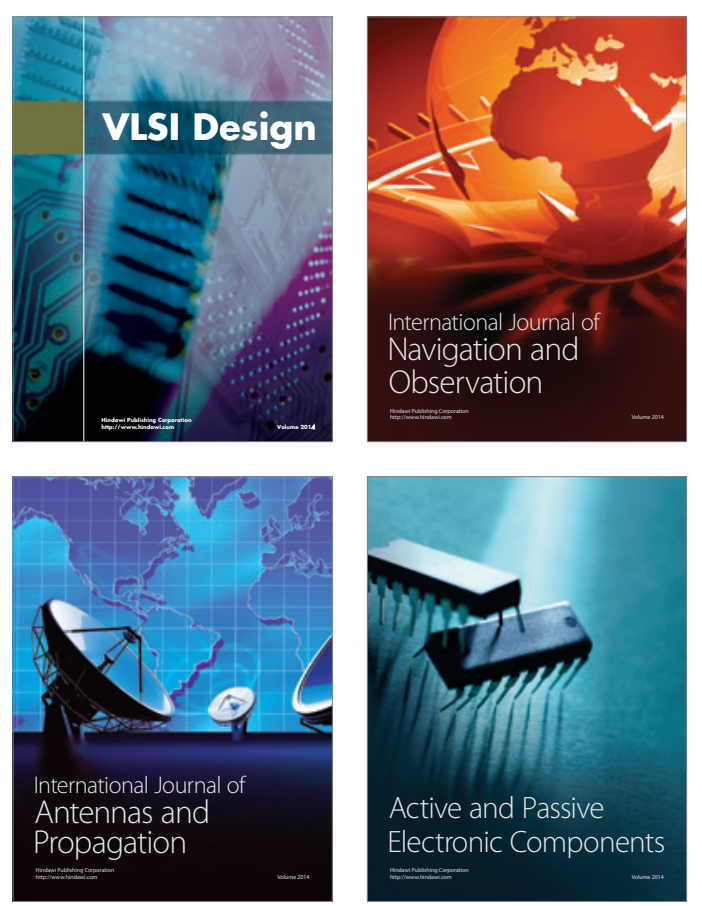
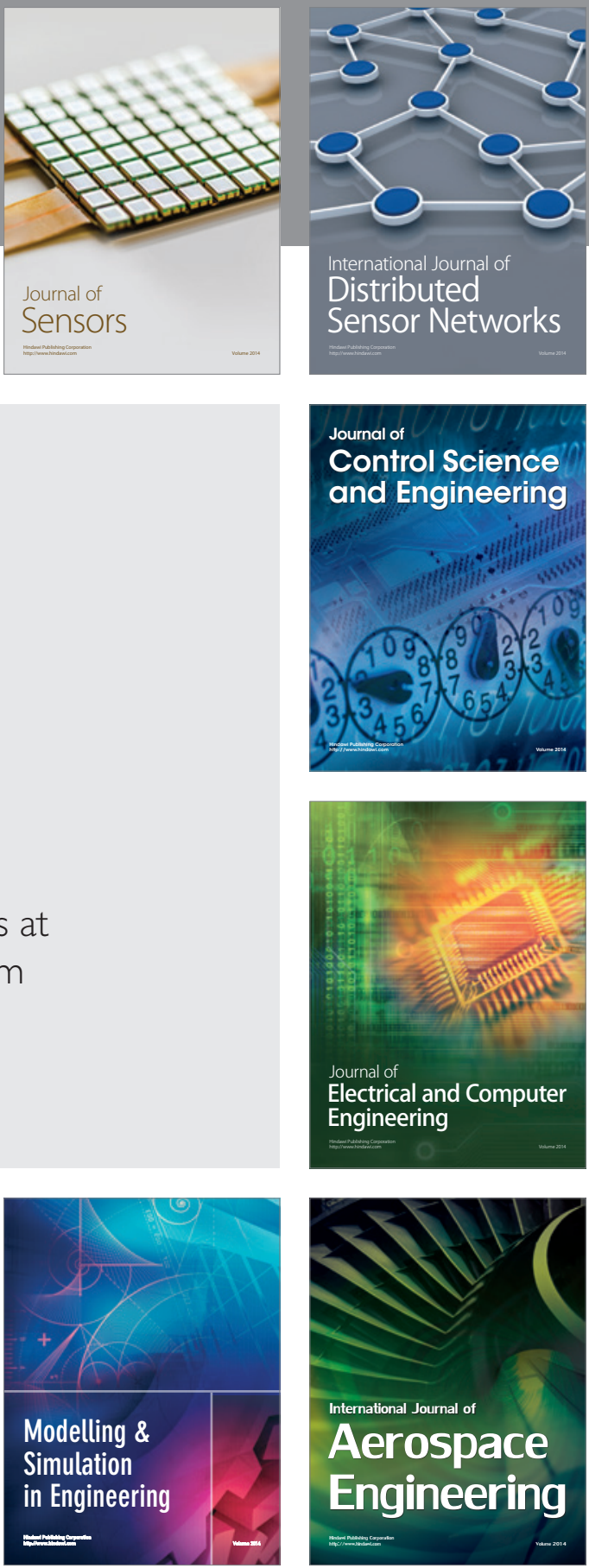

Journal of

Control Science

and Engineering
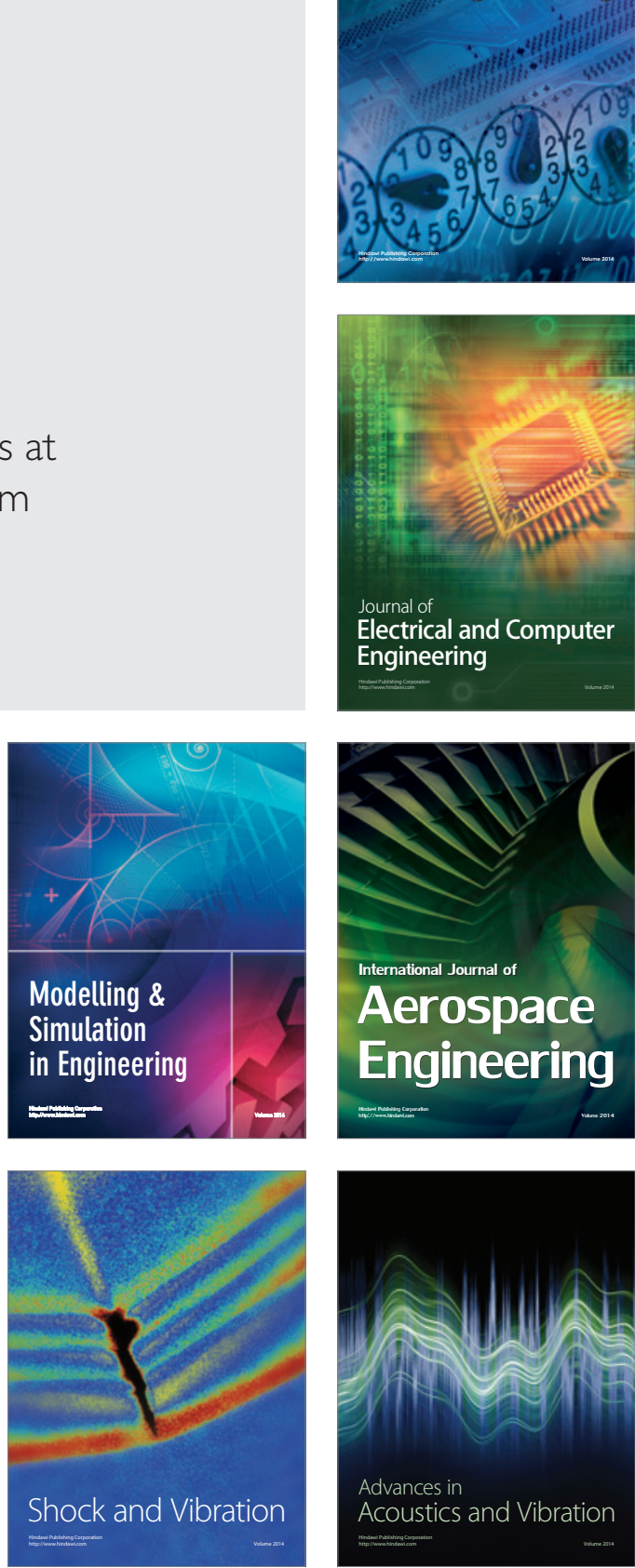